Dear Author,

Please, note that changes made to the HTML content will be added to the article before publication, but are not reflected in this PDF.

Note also that this file should not be used for submitting corrections. 
Ultrasound in Med. \& Biol., Vol. —, No. —, pp. 1-7, 2015 Copyright (C) 2015 World Federation for Ultrasound in Medicine \& Biology Printed in the USA. All rights reserved $0301-5629 / \$$ - see front matter

http://dx.doi.org/10.1016/j.ultrasmedbio.2015.04.005

\title{
PLACENTAL VOLUMETRY BY 2-D SONOGRAPHY WITH A NEW MATHEMATICAL FORMULA: PROSPECTIVE STUDY ON THE SHELL OF A SPHERICAL SECTOR MODEL
}

\author{
Zoltan Kozinszky,* Andrea Surányi ${ }^{\dagger}$ Hajnalka Péics, ${ }^{\ddagger}$ András Molnár, ${ }^{\dagger}$ and Attila PÁl ${ }^{\dagger}$ \\ * Reproductive Medicine, Department of Obstetrics and Gynecology, Huddinge Karolinska University Hospital, Stockholm, \\ Sweden; ${ }^{\dagger}$ Department of Obstetrics and Gynecology, University of Szeged, Szeged, Hungary; and ${ }^{\ddagger}$ Faculty of Civil \\ Engineering, University of Novi Sad, Subotica, Serbia
}

(Received 30 September 2014; revised 12 March 2015; in final form 6 April 2015)

\begin{abstract}
The aim of this study was to determine the utility of a new mathematical model in volumetric assessment of the placenta using 2-D ultrasound. Placental volumetry was performed in a prospective cross-sectional survey by virtual organ computer-aided analysis (VOCAL) with the help of a shell-off method in 346 uncomplicated pregnancies according to STROBE (Strengthening the Reporting of Observational Studies in Epidemiology) guidelines. Furthermore, placental thickness, length and height were measured with the 2-D technique to estimate placental volume based on the mathematical formula for the volume of "the shell of the spherical sector." Fetal size was also assessed by 2-D sonography. The placental volumes measured by 2-D and 3-D techniques had a correlation of 0.86. In the first trimester, the correlation was 0.82 , and later during pregnancy, it was 0.86 . Placental volumetry using "the circle-shaped shell of the spherical sector" mathematical model with 2-D ultrasound technique may be introduced into everyday practice to screen for placental volume deviations associated with adverse pregnancy outcome. (E-mail: kozinszky@gmail.com) (c) 2015 World Federation for Ultrasound in Medicine \& Biology.
\end{abstract}

Key Words: Placenta, Virtual organ computer-aided analysis, Volumetry, 2-D ultrasound technique, 3-D ultrasound technique.

\section{INTRODUCTION}

Fetal birth weight correlates directly with placental weight at birth (Thame et al. 2001, 2004). A small-forgestational-age (SGA) neonate has a placenta with reduced weight and volume compared with the appropriate-for-gestational-age (AGA) counterpart (Derwig et al. 2011; Plasencia et al. 2011), suggesting that adequate fetal growth depends essentially on placental expansion. Placentomegaly can be observed in maternal diabetes, maternal anemia, several fetal malformations (i.e., immune or non-immune hydrops foetalis) and infections (Degani 2006; Kuhlmann and Warsof 1996).

Prediction of intrauterine growth retardation (IUGR) (Derwig et al. 2011; Thame et al. 2004), as well as other adverse outcomes of pregnancy (Gassner et al. 2003; Metzenbauer et al. 2002; Odibo et al. 2011), is a major

Address correspondence to: Zoltan Kozinszky, Reproduktionsmedicin Karolinska, Novum huset Plan 4, 14186 Huddinge, Sweden. E-mail: kozinszky@gmail.com obstetric problem (Salafia et al. 2006). Importantly, an increasing amount of evidence indicates that neonatal birth weight can be predicted by placental volumetry during early to mid-pregnancy (Derwig et al. 2011) using 3-D sonography (Collins et al. 2013; Gassner et al. 2003; Metzenbauer et al. 2002; Odibo et al. 2011; Plasencia et al. 2011, 2012; Rizzo et al. 2012). Placental volume deviation might predict chromosomal abnormalities (Gassner et al. 2003; Metzenbauer et al. 2002), growth retardation (Collins et al. 2013; Hafner et al. 1998, 2006), late-onset pre-eclampsia (Hafner et al. 2006) and other complications of pregnancy (Collins et al. 2013; Hafner et al. 2006; Odibo et al. 2011; Plasencia et al. 2011, 2012; Rizzo et al. 2012; Salafia et al. 2006).

Although 3-D placental volumetry cannot be introduced into everyday practice because it requires special skill, the 2-D technique is simpler and is being used to estimate placental volumes (Azpurua et al. 2010; Costantini et al. 2012; Schwartz et al. 2010). Despite the constant need for estimating placental volume, only one model using the 3-D technique (Schwartz et al. 2012) has been tested so far, and only one previous study on volumetry 
dealt with use of the 2-D technique with a mathematical model (Azpurua et al. 2010). However, placental shape is variable (Abramowicz and Sheiner 2008), and as a consequence, thickness and width measurements of placentas provide contradictory results, which are non-specific (Abramowicz and Sheiner 2008; Salafia et al. 2006) and cannot predict fetal birth weight or perinatal complications satisfactorily (Costantini et al. 2012; Elchalal et al. 2000; Salafia et al. 2006).

Our aim was to determine the applicability of a newly developed mathematical formula based on modeling the placenta as "the shell of the spherical sector" (Figs. 1a-d and 2), which was created by one of the authors (Z.K.). This model was validated by measuring maximal height, length and thickness of a series of placentas by 2-D sonography and calculating volumes with this formula. Correlation analysis of estimated placental volumes (EPV) and measured placental volumes (MPV) was conducted using 3-D technique (Odibo et al. 2011; Pomorski et al. 2012; Rizzo et al. 2012) in normal pregnancies. Another aim of the study was to compare the dependence of placental volume on gestational age.

\section{METHODS}

This prospective observational study comprised a consecutive series of 346 healthy women with singleton pregnancies who underwent ultrasound examination from $9^{+0}$ to $28^{+2}$ wk of gestation at the Department of Obstetrics and Gynecology of the University of Szeged, Hungary. At our unit, all women are routinely offered three ultrasound scans (at 11-13 wk of gestation for nuchal translucency screening, at 16-22 wk to detect any fetal abnormalities and at 24-28 wk to assess fetal growth and development). All women undergoing these scans were invited to participate in this research study between 1 September and 25 December, 2011. The study was approved by the ethics committee of the University of Szeged (Protocol 135/2011). Informed consent was obtained from all participants. Three hundred ninety participants were excluded for pregnancies that resulted in delivery of SGA or large-for-gestational-age neonates (i.e., birth weight below the 10th or above the 90th percentile, respectively); multiple pregnancy; fibroids; enlarged $(>3 \mathrm{~mm})$ nuchal translucency from $11^{+0}$ to $13^{+6} \mathrm{wk}$ of gestation; fetal or neonatal structural/chromosomal

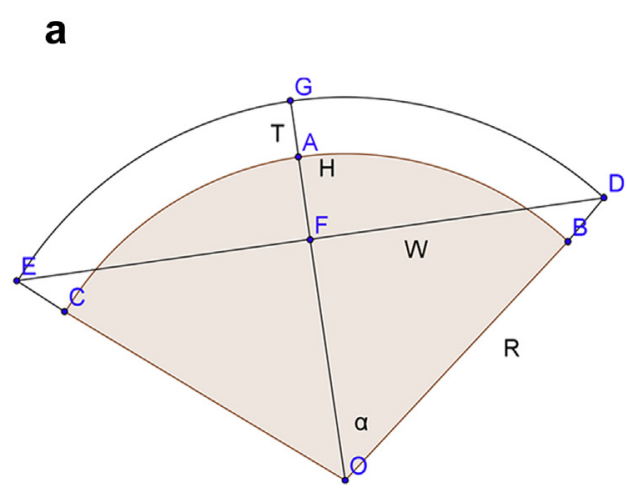

b
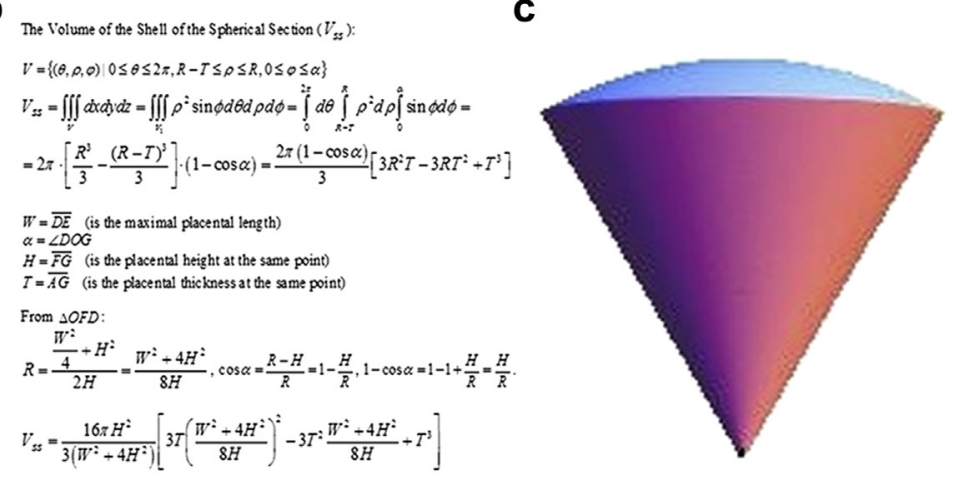

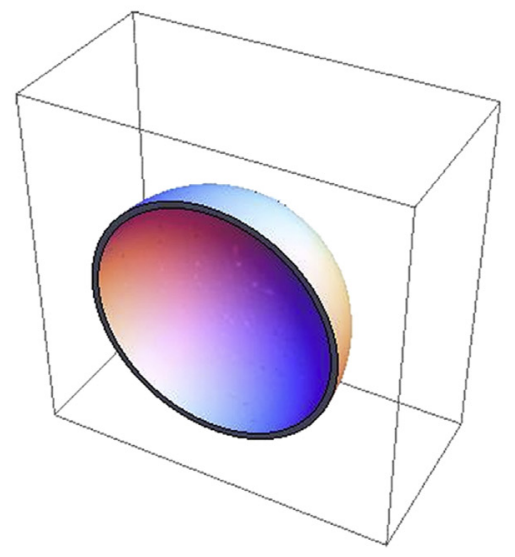

e

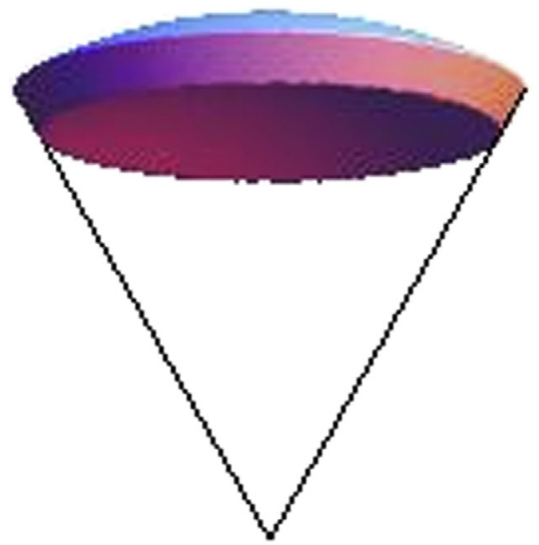

Fig. 1. (a) Section of the spherical sector. (b) Mathematical formula for determining the volume of the shell of the spherical sector $\left(V_{\mathrm{ss}}\right)$. (c) Spherical sector. (d) Shell of a sphere. (e) Shell of a spherical sector. 
a

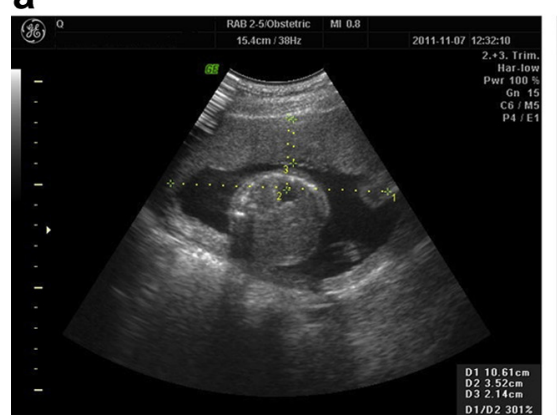

b

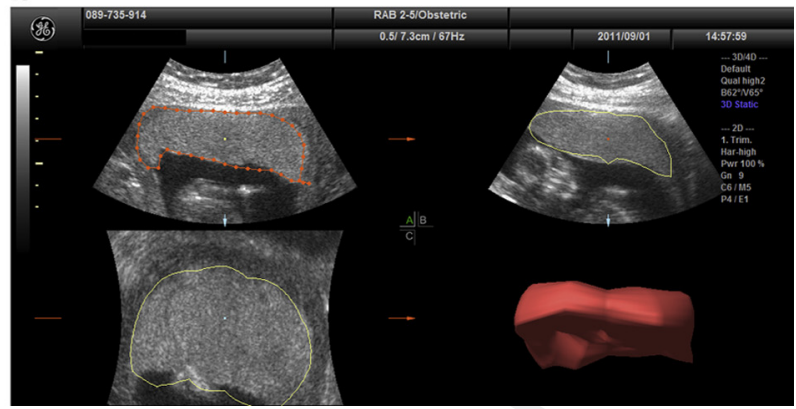

Fig. 2. (a) Placental volumetry parameters used in the 2-D technique: maximal placental length, maximal height of placenta measured perpendicular to the length and thickness at the same point. (b) Placental volumetry using volumetric analysis with the shell-off method by VOCAL 3-D technique. VOCAL = virtual organ computer-aided analysis.

anomalies; amniotic fluid anomalies (i.e., oligohydramnios); inadequate localization (i.e., placenta previa); posterior placenta or functional defect (i.e., placenta abruptio) or structural abnormality of the placenta (i.e., bilobed placenta, placenta with succenturiate lobe); vaginal bleeding; self-reported drug intake; alcohol, caffeine or nicotine abuse; preterm birth; maternal complications during pregnancy such as preeclampsia, diabetes and chronic systematic illness; and failure to sign the consent form. A detailed follow-up was implemented after enrollment (i.e., giving birth at our unit). Inclusion criteria were a singleton pregnancy and an ultrasound scan that was obtained without uterine contractions and captured the entire view of the placenta in one sweep (so that placental length, height and thickness can be visualized in one field [Fig. 2]). The quality of the study was ensured by using a checklist adapted from STROBE (Strengthening the Reporting of Observational Studies in Epidemiology).

\section{Development of the mathematical model for the spherical sector}

Azpurua et al. (2010) worked out a mathematical model for a convex-concave shell formula ("spherical cap") for 2-D volume estimation of the placenta based on measurement of maximal linear length, height and thickness. On the basis of an idea of one of the authors (Z.K.), placental volume was modeled with another mathematical formula - "the shell of the spherical sector"-involving measurement of the same placental parameters, but yielding results other than those of Azpurua et al. (2010). The mathematical formula is used to estimate the placental volume (Fig. 1a-d). A mathematician (H.P.) provided the adaptable mathematical formula for our idea. Maximal placental thickness, height and length were acquired in the image illustrated in Figure 2, where placental thickness and height are perpendicular to placental length.
Volume acquisition by 2-D and 3-D techniques

All 3-D scans and 2-D ultrasound measurements were performed by one specifically trained sonographer (A.S.) to eliminate inter-observer errors. For each patient, sonographic parameters for placental volumes and fetal weights were evaluated three times during a period of maternal breathholding and fetal rest. The average of Q2 three repeated measurements was used for each evaluation. Placentas were assessed with standard 2-D ultrasound, and placental dimensions were determined to obtain the optimal settings for measuring maximal height and thickness at maximal length as linear measurements (Fig. 2). Placental volumes were calculated (estimated placental volumes [EPVs]) on the images where the maximal length was visualized on the longitudinal plane. Where the placenta had substantial thickness at the edge, the caliper was placed midway between the chorionic and basal plates on both edges to measure maximal length. The maximal placental thickness maintaining close proximity to the perpendicular to the placental surface and the line of maximal length was measured. Furthermore, thickness was measured at the maximal length. For laterally located placentas, the transducer was slightly inclined laterally to obtain proper images. Fetal weight was calculated with formula B of Hadlock et al. (1985) after measuring the necessary sonographic parameters (head circumference, abdominal circumference and femur length).

Images for 3-D determination of placental volume (measured placental volume [MPV]) were also obtained after the 2-D scans. A Voluson 730 ultrasound machine (GE Medical Systems, Kretztechnik, Zipf, Austria) equipped with a multifrequency transabdominal convex probe $(2-5 \mathrm{MHz})$ was used to acquire all images. Each sample was examined using 3-D rendering mode, in which the color and gray-value information are processed and combined to create a 3-D image (mode cent; smooth: 4/5; FRQ: low; quality: 16; density: 6; enhance: 16; 
balance: 150; filter: 2 ; actual power: $2 \mathrm{~dB}$; pulse repetition frequency: 0.9) (Rizzo et al. 2012).

A view of the entire placenta was captured by 2-D ultrasound, and the volume box was adjusted to contain the entire placenta. The angle of volume acquisition varied between $45^{\circ}$ and $70^{\circ}$ depending on the size of the placenta. Volume acquisition was obtained at "maximum" speed and took $<10 \mathrm{~s}$, keeping the probe perpendicular to the placental plate. The 3-D static volume box was placed over the entire placenta. The sweep angle was set at a maximum of $70^{\circ}$, and the 3-D volumetric data were stored on a removable hard disk. The longest view of the placenta on plane A was chosen as reference image. The same pre-established instrument settings were used in all cases (Obstetrician/2-3 trimester). Each image was recovered from the disk in succession for processing. The stored volumes marked by outlining the contour of the placenta repeatedly after rotating its image six times by $30^{\circ}$ with manual control to exclude decidua and maternal blood vessels were analyzed using the virtual organ computer-aided analysis (VOCAL) program for 4-D VIEW computer software (GE Medical Systems, Austria, Version 10.4). After rotation had been completed, the placental volume was automatically calculated by the software. The intraobserver correlation coefficients for measuring placental parameters derived by the 2-D and 3-D techniques were excellent ( 0.97 and 0.98 , respectively).

Intra-observer reproducibility, which was calculated after three consecutive measurements of placental parameters for 2-D volumetry, as well as placental volumes, by the single sonographer (A.S.) using the 3-D VOCAL shell-off method, had excellent intra-class coefficients of 0.99 and 0.99 (Fleiss and Cohen 1973), respectively, in 58 participants. Additionally, review of the medical sonographic charts of 1,000 pregnant women revealed that $56.1 \%$ had a placenta partially or totally on the anterior wall of the uterus (anterior or anterior + lateral/fundal), whereas $43.9 \%$ had a placenta on the posterior wall, indicating that in somewhat less than half of pregnancies, this technical problem in measurement of placental volume is encountered.

\section{Statistical analyses}

Regression curve analyses were performed for both first- and second-trimester placental volume data using SPSS software (Version 20, IBM, Armonk, NY, USA) to optimize the fitting of curves to our plot. Curve estimation models were as follows: linear, logarithmic, inverse, quadratic, power, compound, S-shaped curve, logistic, growth and exponential relations (Freedman 2005). A $p$ value $<0.05$ was considered to indicate statistical significance. The associations between placental volumes measured with the 2-D and 3-D techniques and estimated fetal weights were determined with Pearson's correlation. A.S. was blinded to the mathematical model and obtained technical instructions for the sonographic measurements, but all statistical analyses were performed by Z.K.

\section{RESULTS}

Placental volume was measured by volume analysis with the shell-off method using 3-D VOCAL technique and estimated with the help of the mathematical formula measuring length, height and thickness of a total of 346 pregnancies. In addition, fetal weight was estimated from the measurements of head circumference, abdominal circumference and femur length at the same time.

The median age of enrolled pregnant women was 32 y (range: $18-43 \mathrm{y}$ ), and the median gestational age was 19 wk (range: 9-28.3 wk). The data sets of measured placental volumes (MPVs) determined with the shelloff method using 3-D technique and estimated by the mathematical formula on "the shell of the spherical sector" (EPVs) were plotted (Fig. 3). The correlation between MPVs (mean: $158 \mathrm{~cm}^{3}$, range: $31-611 \mathrm{~cm}^{3}$ ) and EPVs (mean: $189 \mathrm{~cm}^{3}, 11-922 \mathrm{~cm}^{3}$ ) was highly significant $(p<0.001)$ and strong $(r=0.86)$. The volumes calculated from the "spherical cap" model yielded a lower, but yet strong and significant correlation ( $r=0.80, p<0.001$ ) (Azpurua et al. 2010).

The correlation between MPVs and EPVs was significant $(r=0.82, p<0.05)$ during the first trimester (between $9^{+0}$ and $11^{+6} \mathrm{wk}, 29$ cases) and strong ( $r=$ 0.86 ) in the second trimester (between $12^{+0} \mathrm{wk}$ and $28^{+2}$ wk, 317 cases).

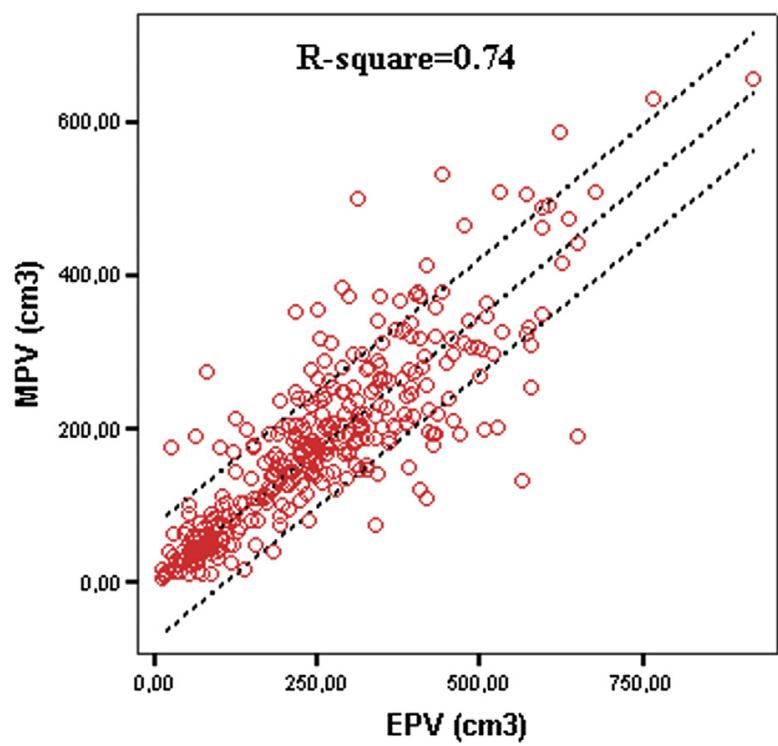

Fig. 3. MPV versus EPV. MPV = measured placental volume; $\mathrm{EPV}=$ estimated placental volume. 
Regression curve analyses revealed that all of the curve estimation models (linear, logarithmic, inverse, quadratic, power, compound, S-shaped curve, logistic, growth and exponential) can be significantly fit into the plotted data, but EPVs were related most significantly to gestational age in the case of the S-shaped curve $(p$ $<0.001, r=0.758)$ when the mathematical model was applied. In addition, the exponential $(p<0.001, r=$ $0.717)$ regression also provided robust dependency. The linear correlation appeared to provide a less appropriate, but yet significant regression both for EPVs $(p<0.001$, $r=0.572)$ and for MPVs $(p<0.001, r=0.493)$ (Figs. 4 and 5).

Estimated fetal weight (EFW) significantly correlated both with MPVs $(r=0.66, p<0.001)$ and EPVs $(r=0.72, p<0.001)$. In our data set, the EPVs that were calculated based on the "spherical cap" model also revealed a significant correlation $(r=0.71, p<$ $0.001)$. However, there was a reduced, but significant correlation between EFW and placental thickness $(r=0.43$, $p<0.001)$ and length $(r=0.66, p<0.001)$.

\section{DISCUSSION}

An increasing amount of evidence has been published on the clinical significance of placental weight at birth and placental weight relative to fetal birth weight with respect to fetomaternal diseases, obstetric and neonatal outcomes, perinatal morbidity/mortality and childhood growth and development (Almog et al. 2011). In addition, a disproportionally small placenta is characteristic of fetal death (Haavaldsen et al. 2013; Hasegawa et al. 2011), and growth-retarded fetuses often have small placentas (Almog et al. 2011). Unfavorable placental and fetal growth in utero predisposes to

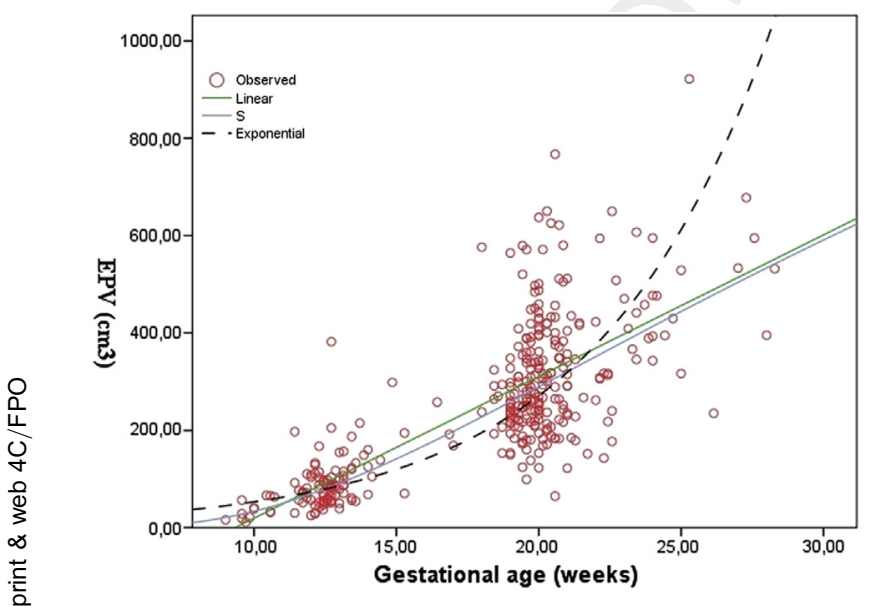

Fig. 4. EPV versus gestational age. The graphs depict the linear, $S$-shaped curve and exponential trend lines. EPV = estimated placental volume.

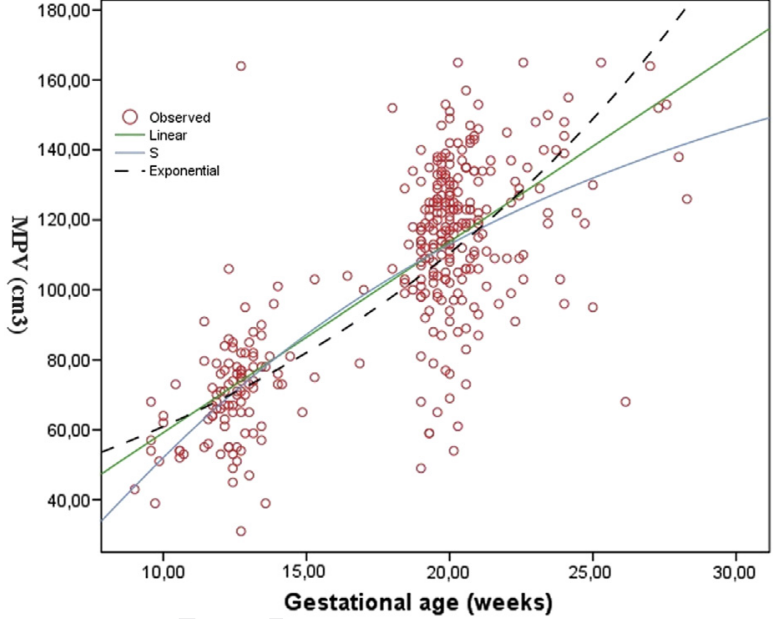

Fig. 5. MPV versus gestational age. The graphs depict the linear, S-shaped curve and exponential trend lines. MPV = measured placental volume.

diabetes, coronary heart disease and hypertension at subsequent ages (Barker and Thornburg 2013).

Salafia et al. (2006) suggested that a placenta that is large relative to fetal weight might represent placental insufficiency with reduced ability to maintain fetal growth. Thus, sonographic estimation of placental growth in addition to fetal growth is an important perinatal predictive indicator (Gassner et al. 2003; Hafner et al. 1998; Odibo et al. 2011; Salafia et al. 2006; Thame et al. 2001, 2004) and might be an adjunct modality in determining uteroplacental flow (Rotmensch et al. 1994). These facts underline the necessity of routine placental volumetry in everyday practice. Magnetic resonance imaging (Derwig et al. 2011) and the 3-D ultrasound technique (Almog et al. 2011; Collins et al. 2013; Gassner et al. 2003; Metzenbauer et al. 2002; Odibo et al. 2011; Plasencia et al. 2011, 2012; Rizzo et al. 2012; Salafia et al. 2006) are highly precise methods, but are time consuming and expensive and require considerable expertise.

We render a new mathematical formula that is based on parameters that can easily be measured by 2-D sonographic technique and correlates highly $(r=0.86)$ with placental volumes measured using 3-D technique. In very special cases in which the entire placenta is not visible in one ultrasound section, however, both edges, thickness and height, can be visualized; placental volume can be calculated with 2-D technique, but cannot be measured with the 3-D VOCAL technique. "The shell of the spherical sector" is a model representing a round placenta adhering to the internal surface of a spherelike uterus. However, placental shape varies from elliptical to other irregular shapes, which may result in under- or overestimated volumes. Our new formula yields 
a higher correlation than another 2-D mathematical formula ( $r=0.80)$ based on a "spherical cap" reported by Azpurua et al. (2010), which is characterized by a portion of a sphere cut off by a plane. We believe that the model we describe may be of interest in the future for the prediction and diagnosis of maternal/fetal complications and the follow-up of small placentas up to the first half of the third trimester. Large placentas could be followed up only until the late second trimester, as their length extends to the maximal sonographic field.

In addition, we compared estimates of placental volume with measurements, and the "spherical cap" mathematical formula revealed a good correlation with measured placental volumes and weight after delivery $(r=0.80)$. Moreover, our method was verified only by comparing 2-D and 3-D estimated volume data sets. U1trasound estimates tend to have an inaccuracy of $\pm 10 \%-20 \%$. The placental volumes estimated on the basis of our model correlated significantly with the actual estimated fetal weights, in agreement with studies describing a strong correlation between placental and fetal weights in both the first and second trimesters and at delivery as well (Almog et al. 2011; Derwig et al. 2011; Hafner et al. 1998; Thame et al. 2001).

Our results suggest that placental growth follows an S-shape (Kozinszky and Surányi 2012) or an exponential line between 9 and 29 wk of gestation, which is in accordance with other findings of S-shaped or nearly exponential placental birth weight nomograms in the third trimester (Almog et al. 2011; Thame et al. 2001, 2004). Placental volume increases from a median of $22 \mathrm{~cm}^{3}$ at about 9 wk of gestation to $490 \mathrm{~cm}^{3}$ at about $28 \mathrm{wk}$ of gestation, in agreement with a previous study (Derwig et al. 2011).

One of the limitations of the method is that the section of the ultrasound probes is $45^{\circ}-70^{\circ}$, which limits the measurable placental length; this means that placental growth can be studied mainly until the beginning of the third trimester (Hafner et al. 1998). This is in line with the fact that the lateral growth of the chorionic disc is, to a great extent, completed by 30 to $32 \mathrm{wk}$ of gestation (Craven et al. 2000). Another limitation of the 2-D and 3-D techniques for measurement of the placenta located on the posterior uterine wall is that the echogenic shadow of the fetus might disturb the imaging of the placenta and make placental volumetry impossible in an increasing proportion as gestation advances from the first to the third trimester.

Two-dimensional sonographic placental volumetry might be more useful as a screening tool in daily routine in the follow-up of the rate and pattern of placental growth of growth-restricted fetuses and fetuses of pregnant women with pre-eclampsia and diabetes, providing information additional to that provided by fetal biometry alone or fetal biometry and 2-D Doppler flowmetry in combination (Derwig et al. 2011; Odibo et al. 2011). Our study might provide evidence of the possible applicability of our mathematical model to placental volumetry; however, it should be tested in pathologic pregnancies before it is introduced as a general screening test in the first and second trimesters.

Acknowledgments-Special thanks to Gabor Gasparfor linguistic correction of the article.

\section{REFERENCES}

Abramowicz JS, Sheiner E. Ultrasound of the placenta: A systematic approach. Part I: Imaging. Placenta 2008;29:225-240.

Almog B, Shehata F, Aljabri S, Levin I, Shalom-Paz E, Shrim A Placenta weight percentile curves for singleton and twins deliveries. Placenta 2011;32:58-62.

Azpurua H, Funai EF, Coraluzzi LM, Doherty LF, Sasson IE, Kliman M, Kliman HJ. Determination of placental weight using twodimensional sonography and volumetric mathematic modeling. Am J Perinatol 2010;27:151-155.

Barker DJ, Thornburg KL. Placental programming of chronic diseases, cancer and lifespan: A review. Placenta 2013;34:841-845.

Collins SL, Stevenson GN, Noble JA, Impey L. Rapid calculation of standardized placental volume at 11 to 13 weeks and the prediction of small for gestational age babies. Ultrasound Med Biol 2013;39: 253-260.

Costantini D, Walker M, Milligan N, Keating S, Kingdom J. Pathologic basis of improving the screening utility of 2-dimensional placental morphology ultrasound. Placenta 2012;33:845-849.

Craven CM, Zhao L, Ward K. Lateral placental growth occurs by trophoblast cell invasion of decidual veins. Placenta 2000;21:160-169.

Freedman David A. Statistical models: Theory and practice. Cambridge: Cambridge University Press; 2005.

Degani S. Sonographic findings in fetal viral infections: A systematic review. Obstet Gynecol Surv 2006;61:329-336.

Derwig IE, Akolekar R, Zelaya FO, Gowland PA, Barker GJ, Nicolaides KH. Association of placental volume measured by MRI and birth weight percentile. J Magn Reson Imaging 2011;34: 1125-1130.

Elchalal U, Ezra Y, Levi Y, Bar-Oz B, Yanai N, Intrator O, Nadjari M. Sonographically thick placenta: A marker for increased perinatal risk-A prospective cross-sectional study. Placenta 2000;21: 268-272.

Fleiss JL, Cohen J. The equivalence of weighted kappa and the intraclass correlation coefficient as measures of reliability. Educ Psychol Meas 1973;33:613-619.

Gassner R, Metzenbauer M, Hafner E, Vallazza U, Philipp K. Triploidy in a twin pregnancy: Small placental volume as an early sonographical marker. Prenat Diagn 2003;23:16-20.

Haavaldsen C, Samuelsen SO, Eskild A. Fetal death and placental weight/birthweight ratio: A population study. Acta Obstet Gynecol Scand 2013;92:583-590.

Hadlock F, Harrist R, Sharman R, Deter R, Park S. Estimation of fetal weight with the use of head, body and femur measurements: A prospective study. Am J Obstet Gynecol 1985;151:333-337.

Hafner E, Metzenbauer M, Höfinger D, Stonek F, Schuchter K, Waldhör T, Philipp K. Comparison between three-dimensional placental volume at 12 weeks and uterine artery impedance/notching at 22 weeks in screening for pregnancy-induced hypertension, preeclampsia and fetal growth restriction in a low-risk population. U1trasound Obstet Gynecol 2006;27:652-657.

Hafner E, Philipp T, Schuchter K, Dillinger-Paller B, Philipp K, Bauer P. Second-trimester measurements of placental volume by threedimensional ultrasound to predict small-for-gestational-age infants. Ultrasound Obstet Gynecol 1998;12:97-102.

Hasegawa J, Arakawa K, Nakamura M, Matsuoka R, Ichizuka K, Katsufumi O, Sekizawa A, Okai T. Analysis of placental weight 
centiles is useful to estimate cause of fetal growth restriction. J Obstet Gynaecol Res 2011;37:1658-1665.

Kozinszky Z, Surányi A. Re: Placental volumes measured by 3dimensional ultrasonography in normal pregnancies from 12 to 40 weeks' gestation. J Ultrasound Med 2012;31:2044-2045.

Kuhlmann RS, Warsof S. Ultrasound of the placenta. Clin Obstet Gynecol 1996;39:519-534.

Metzenbauer M, Hafner E, Schuchter K, Philipp K. First-trimester placental volume as a marker for chromosomal anomalies: Preliminary results from an unselected population. Ultrasound Obstet Gynecol 2002;19:240-242.

Odibo AO, Goetzinger KR, Huster KM, Christiansen JK, Odibo L, Tuuli MG. Placental volume and vascular flow assessed by 3-D power Doppler and adverse pregnancy outcomes. Placenta 2011;32: 230-234.

Plasencia W, Akolekar R, Dagklis T, Veduta A, Nicolaides KH. Placental volume at 11-13 weeks' gestation in the prediction of birth weight percentile. Fetal Diagn Ther 2011;30:23-28.

Plasencia W, González Dávila E, Tetilla V, Padrón Pérez E, García Hernández JA, González González NL. First-trimester screening for large-for-gestational-age infants. Ultrasound Obstet Gynecol 2012;39:389-395.

Pomorski M, Zimmer M, Florjanski J, Michniewicz J, Wiatrowski A, Fuchs T, Milnerowicz-Nabzdyk E. Comparative analysis of placental vasculature and placental volume in normal and IUGR pregnancies with the use of three-dimensional Power Doppler. Arch Gynecol Obstet 2012;285:331-337.

Rizzo G, Capponi A, Pietrolucci ME, Aiello E, Arduini D. First trimester placental volume and three dimensional power Doppler ultrasonography in type I diabetic pregnancies. Prenat Diagn 2012;32: 480-484.

Rotmensch S, Liberati M, Luo JS, Kliman HJ, Gollin Y, Bellati U, Hobbins JC, Copel JA. Color Doppler flow patterns and flow velocity waveforms of the intraplacental fetal circulation in growth-retarded fetuses. Am J Obstet Gynecol 1994;171: $1257-1264$.

Salafia CM, Charles AK, Maas EM. Placenta and fetal growth restriction. Clin Obstet Gynecol 2006;49:236-256.

Schwartz N, Coletta J, Pessel C, Feng R, Timor-Tritsch IE, Parry S, Salafia CN. Novel 3-dimensional placental measurements in early pregnancy as predictors of adverse pregnancy outcomes. J Ultrasound Med 2010;29:1203-1212.

Schwartz N, Wang E, Parry S. Two-dimensional sonographic placental measurements in the prediction of small-for-gestational-age infants. Ultrasound Obstet Gynecol 2012;40:674-679.

Thame M, Osmond C, Bennett F, Wilks R, Forrester TE. Fetal growth is directly related to maternal anthropometry and placental volume. Eur J Clin Nutr 2004;58:894-900.

Thame M, Osmond C, Wilks R, Bennett FI, Forrester TE. Secondtrimester placental volume and infant size at birth. Obstet Gynecol 2001;98:279-283. 